\title{
Single- and multiple-set resistance training improves skeletal and respiratory muscle strength in elderly women
}

This article was published in the following Dove Press journal:

Clinical Interventions in Aging

16 October 2014

Number of times this article has been viewed

\author{
Odilon Abrahin ${ }^{1-3}$ \\ Rejane P Rodrigues ${ }^{1-3}$ \\ Vanderson C Nascimento ${ }^{3}$ \\ Marzo E Da Silva- \\ Grigoletto ${ }^{1,4}$ \\ Evitom C Sousa ${ }^{3}$ \\ Anderson C Marçal ${ }^{1,2}$ \\ 'Department of Physical Education, \\ Federal University of Sergipe, \\ Sergipe, Brazil; ${ }^{2}$ Center of Research \\ in Intracellular Signaling, Department \\ of Morphology, Federal University \\ of Sergipe, Sergipe, Brazil; ${ }^{3}$ Laboratory \\ of Resistance Exercise and Health, \\ Sports Department, University of Pará \\ State, Belem, Brazil; ${ }^{4}$ Scientific Sport, \\ Sergipe, Brazil
}

Introduction: Aging involves a progressive reduction of respiratory muscle strength as well as muscle strength.

Purpose: Compare the effects of resistance training volume on the maximum inspiratory pressure (MIP), maximum expiratory pressure (MEP), functional performance, and muscle strength in elderly women.

Methods: Thirty elderly women were randomly assigned to a group performing either single sets (1-SET) or three sets (3-SET) of exercises. The sit-to-stand test, MIP, MEP, and muscle strength were assessed before and after 24 training sessions. Progressive resistance training was performed two times per week for a total of 8-12 repetitions, using the main muscle groups of the upper and lower limbs.

Results: The main results showed that the participants significantly increased their MEP $(P<0.05$; 1-SET: 34.6\%; 3-SET: 35.8\%) and MIP ( $P<0.05$; 1-SET: $13.7 \%$; 3-SET: $11.2 \%)$ Both groups also improved in the sit-to-stand test ( $P<0.05 ; 1$-SET: $10.6 \%$; 3 -SET: $17.1 \%)$. After 24 training sessions, muscle strength also significantly increased $(P<0.0001 ; 40 \%-80 \%)$ in both groups. An intergroup comparison did not show any statistically significant differences between the groups in any of the parameters analyzed.

Conclusion: Single- and multiple-set resistance training programs increased MIP, MEP, muscle strength, and sit-to-stand test performance in elderly women after 24 sessions of training. In conclusion, our results suggested that elderly women who are not in the habit of physical activity may start with single-set resistance training programs as a short-term strategy for the maintenance of health.

Keywords: resistance exercise, maximum inspiratory pressure, maximum expiratory pressure, elderly

\section{Introduction}

Aging can be defined as a process of biological modifications involving a gradual reduction in the capacity to adapt, and an increase in vulnerability to countless health issues such as chronic noncommunicable diseases and musculoskeletal disorders. According to the World Health Organization, by 2050, the number of elderly people (older than 60 years) is estimated to reach 2 billion, corresponding to approximately $22 \%$ of the global population. ${ }^{1}$

In general terms, sedentary aging involves a reduction in physical capacity, in association with functional deficits, such as reduced levels of respiratory muscle strength and muscle strength, reduced cardiorespiratory capacity, and reduced mobility, all of which make completing daily activities more difficult. ${ }^{2-4}$ A recent study established a strong association between poor physical fitness and respiratory disorders. ${ }^{4}$ 
Dysfunction in the respiratory muscles can lead to hyperventilation, reduction in exercise tolerance, and even respiratory insufficiency; also, it is associated with an increase in morbidity and mortality rates. ${ }^{4,5}$ The evaluation of respiratory muscle strength is of great clinical importance and can be measured by static and dynamic maneuvers. ${ }^{6-9}$ Static maneuvers infer maximal inspiratory pressure (MIP) and maximal expiratory pressure (MEP). These parameters, evaluated through the mouth, reflect the pressure that is being generated by the action of the respiratory muscles. ${ }^{6-9}$

Specific respiratory muscle training has had significant effects on the respiratory muscle strength and endurance of athletes. ${ }^{10}$ However, few scientific studies suggest that resistance training can be used for improving respiratory muscle strength. ${ }^{11,12}$ To the best of our knowledge, no study has investigated the chronic effect of resistance training (RT) volume on the respiratory muscles in elderly women. Therefore, the aim of the present study was to compare the effects of RT volume on the MIP, MEP, functional performance, and muscle strength in elderly women.

\section{Methods}

\section{Subjects}

The inclusion criteria required the women to be nonsmokers and between 60 and 80 years of age, with previous experience of RT (minimum 6 months, uninterrupted), but without participation in any type of physical exercise during the preceding 3 months. Volunteers who had any type of musculoskeletal, cardiovascular, or neurological disorder that complicated RT were excluded, as were women who could not complete the 24 training sessions.

Thirty elderly women from the local community agreed to participate in this study. The volunteers were randomly divided into two groups: 1-SET ( $n=15)$, and 3-SET $(n=15)$. By the end of the study, 19 participants had successfully completed the study protocol (1-SET, $n=11 ; 3-\mathrm{SET}, \mathrm{n}=8$ ); the reasons for dropout were not related to adverse events associated with the protocol, and the participants who did not complete 24 training sessions or who missed four consecutive sessions were excluded (Figure 1).

All participants presented a medical certificate and signed the Termo de Consentimento Livre e Esclarecido (informed consent form) for their participation in this research. The study was approved by the Research Ethics Committee of Universidade da Amazônia (CAAE [certificate of presentation for ethical consideration] number: 12941013.5.0000.5173), in accordance with both the regulations of Resolution 196/96 of Conselho Nacional de Pesquisa Envolvendo Seres Humanos (National Council on Ethics in Human Research) and the Declaration of Helsinki.

\section{Procedures}

The participants initially performed two sessions of training in order to learn the exercises. Thereafter, they attended an additional four sessions. During the first session, anthropometry and functional performance were assessed with the sit-to-stand test. On the second visit, MIP and MEP were

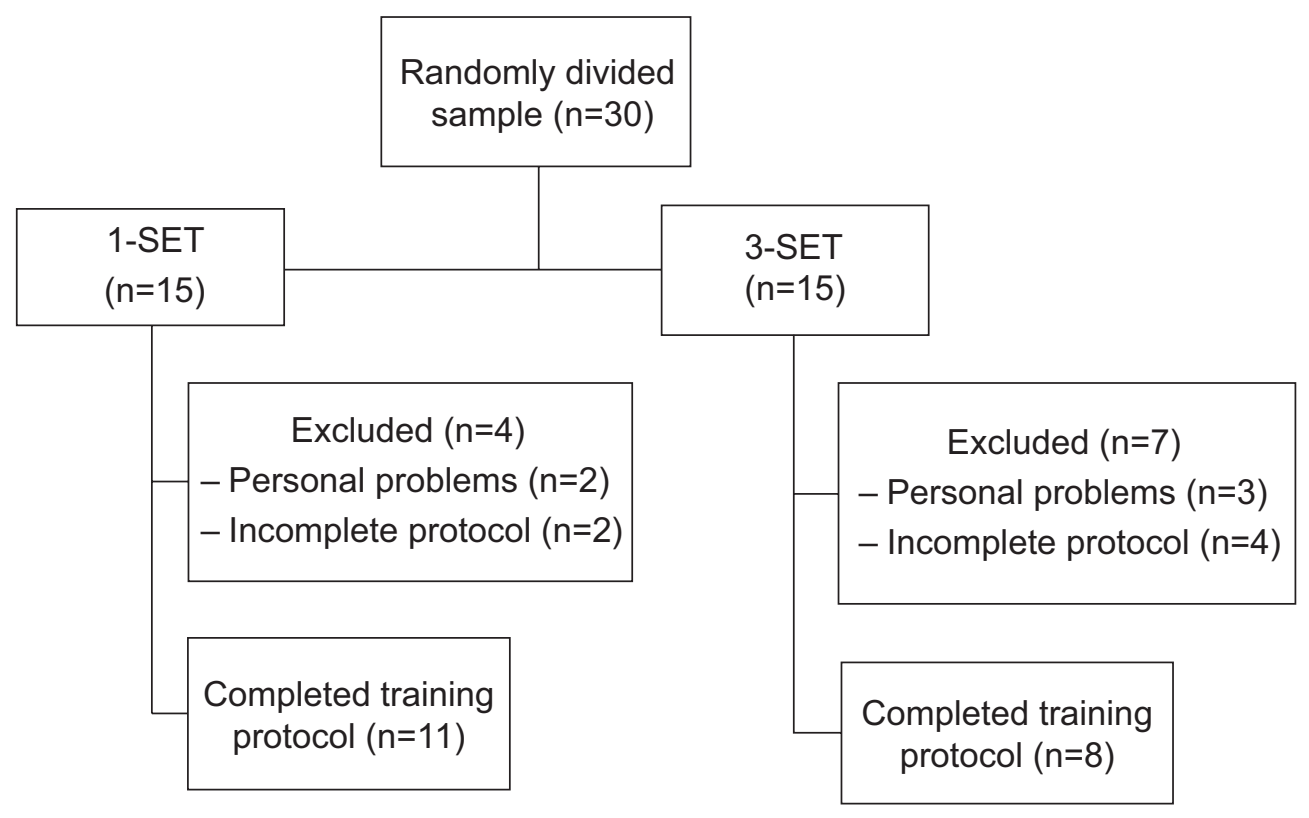

Figure I Flowchart of the volunteer selection process in different stages of the study. Abbreviations: I-SET, single set; 3-SET, three sets. 
assessed. On the third and fourth visits, testing and retesting 10 maximum repetitions (MR) were conducted. After 24 sessions of progressive RT, the tests were reassessed in the same sequence and by the same evaluator.

\section{Sit-to-stand in 30 seconds}

The assessment was started using a chair with a height of $43 \mathrm{~cm}$; the volunteer sat in the middle of the seat, with a straight spinal column, feet resting on the ground and arms crossed against the chest. When signaled, the volunteer was encouraged to fully sit and stand as many times as possible in 30 seconds. The test was performed as described by Rikli and Jones. ${ }^{13}$ All patients were familiarized with the testing, and the best time of two attempts was used.

\section{Anthropometry}

For the measurement of waist and abdominal circumference, a flexible metallic tape measure with precise $0.1 \mathrm{~cm}$ markings was used (Sanny, São Paulo, Brazil). Body fat percentages were estimated using a Skinfold Caliper (Harpenden, London, UK) and an analog scale with a stadiometer, having a $180 \mathrm{~kg}$ capacity in $100 \mathrm{~g}$ divisions. Skinfold thicknesses were measured according to the standard set by the Durnin and Womersley ${ }^{14}$ equation for elderly women, and the Siri ${ }^{15}$ equation. All measures were taken by an International Society for the Advancement of Kinanthropometry Level 3 anthropometrist with at least 10 years of experience, with reliability tested by technical error of measurement, as proposed by Norton and Olds, ${ }^{16}$ being lower than $2 \%$.

\section{Maximum inspiratory pressure and maximum expiratory pressure}

MIP and MEP were measured in stages using an analog manometer (MV 300; Wika, São Paulo, Brazil), with a pressure range of $-300 \mathrm{cmH}_{2} \mathrm{O}$ to $+300 \mathrm{cmH}_{2} \mathrm{O}$. Both MIP and MEP measurements were performed with a plastic tube $3 \mathrm{~cm}$ in diameter and $15 \mathrm{~cm}$ long, with a plastic flanged mouthpiece and a small air leak $7.5 \mathrm{~cm}$ from the mouthpiece. The small leak in the tube was necessary to prevent generation of high buccal pressures. In addition, subjects were required to hold their cheeks with one hand during the performances. Verbal encouragement was given to the subjects during testing, to ensure that motivation levels remained high. Measurements followed the recommendations of the American Thoracic Society/European Respiratory Society ${ }^{6}$ and were conducted three times for each participant by an experienced evaluator. The highest value was used for the analysis. In cases where a difference exceeding 10\% was found between repeated measurements, the exercise was repeated to obtain the highest measurement, but not necessarily the final one. ${ }^{6,7}$

\section{Muscle strength (IO MR)}

Muscle strength was assessed by MR tests in accordance with American College of Sports Medicine ${ }^{17}$ recommendations, using bench press, deadlift, unilateral rowing, and standing calf raise. All participants underwent two test sessions, with an interval of $>48$ hours between sessions. The test and retesting were conducted in the same order, to minimize possible errors in the $10 \mathrm{MR}$ tests. Each session comprised one set of warm-ups, with an estimated $50 \%$ load on the first attempt and up to three consecutive attempts, in order to manage a load of $10 \mathrm{MR}$. The recovery interval between attempts was 5 minutes.

\section{Resistance training program}

The 1-SET $(n=11)$ and 3-SET $(n=8)$ groups conducted RT two times per week for 12 weeks, with a minimum of 48 hours between sessions. All sessions were conducted in the Laboratory of Resistance Training and Health, and participants were instructed to maintain their daily activities and normal eating habits throughout the 24 training sessions.

Both groups conducted the following exercises: bench press, deadlift, unilateral rowing, standing calf raise, and lower abdominal exercise. Such exercises involve the major muscle groups of the upper and lower limbs and simulate the basic movements of daily activities. All exercises were performed in the same order, with an intensity of 8-12 MR. The participants were instructed to adjust their training load in order to guarantee submaximal/maximal strength between 8 and $12 \mathrm{MR}$. The following characteristics were observed in the technical performance of the exercises: tendency for concentric muscle failure and reduction in rhythm, apnea, and isometry. If the volunteers were able to complete 12 repetitions of an exercise in each of two consecutive training sessions, the loads were increased by $5 \% .{ }^{17}$ All sessions were supervised and involved small groups of up to six participants, to ensure that each exercise technique was performed correctly, safely, and to the appropriate intensity.

In each experimental group (1-SET and 3-SET), the type of training method alternated by segment. ${ }^{17}$ Other RT variables, including intensity, repetitions, speed, order, interval, and weekly frequency were the same for both groups; the only difference between the groups was the number of sets (Table 1). 
Table I Resistance training variables used in this study

\begin{tabular}{lll}
\hline Variables & I-SET & 3-SET \\
\hline Intensity & $60 \%-80 \%$ of $10 \mathrm{MR}$ & $60 \%-80 \%$ of I0 MR \\
Repetitions & $8-12$ & 2 seconds of eccentric and 2 seconds of concentric \\
Speed & 90 seconds & 2 seconds' eccentric and concentric \\
Interval between exercises & - & 2 times per week \\
Interval between sets & 5 exercises seconds \\
Frequency & I set per exercise & 2 times per week \\
Number of exercises & 3 sets per exercise \\
Set &
\end{tabular}

Abbreviations: I-SET, single set; 3-SET, three sets; MR, maximum repetitions.

\section{Training sessions}

The single- and multiple-set protocols had a maximum duration of 20 minutes and 50 minutes, respectively. At the beginning of each session, the individuals performed a warm-up of approximately 5 minutes in duration. The warm-up involved exercises for the upper and lower limbs: shoulder adduction and abduction, torso rotation, hip flexion and extension, knee flexion and extension, and ankle flexion and extension.

\section{Variable reproducibility}

The test and retest for $10 \mathrm{MR}$ was conducted using an interval of 48 hours. The intraclass correlation coefficients were 0.90 , 0.94, 0.77, and 0.82 for bench press, deadlift, rowing, and standing calf raise, respectively. All volunteers were familiar with all the tests, as they had participated in other projects within the laboratory during the previous semester.

\section{Statistical analysis}

Traditional statistical methods were used to calculate mean \pm standard deviation. Sample normality was calculated using the Shapiro-Wilk test. The effect of the different interventions single- versus multiple-set (independent variables) on MIP, MEP, and muscular strength (dependent variables) were analyzed by means of ANOVA $(2 \times 2)$. A Sidak correction was used to adjust the $P$-value regarding the number of contrasts performed, and a $P<0.05$ criterion was used to establish statistical significance. Reproducibility was assessed using an intraclass correlation coefficient, and effect size was calculated for paired variables. The SPSS 18 package (IBM Corp., Armonk, NY) for Windows was used for all statistical tests.

On the basis of a pilot study (four subjects), as well as available literature, a power analysis was performed to determine the appropriate number of subjects. Eight subjects (per group) were required to detect a minimum difference of $12 \mathrm{cmH}_{2} \mathrm{O}$ for MIP and $10 \mathrm{cmH}_{2} \mathrm{O}$ for MEP (Granmo 5.2 for Windows; IMIM, Barcelona, Spain), which would be required to achieve $80 \%$ statistical power.

\section{Results}

The characteristics of the women in this study are presented in Table 2. There were no significant differences in age, weight, body mass index, body fat percentage, or waist and abdomen circumferences between the groups at the beginning of the study. Furthermore, these variables were not significantly different after training.

The 1-SET and 3-SET groups significantly improved $(P<0.05)$ in the MEP (pre: $79.5 \pm 20.5 \mathrm{cmH}_{2} \mathrm{O}$; post: $105.5 \pm 24.6$ $\mathrm{cmH}_{2} \mathrm{O}$; effect size: 1.27) and (pre: $76.3 \pm 10.9 \mathrm{cmH}_{2} \mathrm{O}$;

Table 2 Characteristics of elderly women before and after 24 sessions of training; women performing a single set (I-SET, $\mathrm{n}=\mathrm{I}$ I) and those performing three sets (3-SET, $n=8)$

\begin{tabular}{|c|c|c|c|c|}
\hline & \multicolumn{2}{|l|}{ Before } & \multicolumn{2}{|l|}{ After } \\
\hline & I-SET & 3-SET & I-SET & 3-SET \\
\hline Age & $67.1 \pm 3.7$ & $69.4 \pm 6.0$ & & \\
\hline Body mass (kg) & $65.1 \pm 12.1$ & $63.9 \pm 8.1$ & $65.7 \pm 11.9$ & $64.3 \pm 8.3$ \\
\hline Height $(\mathrm{cm})$ & $153 \pm 0.1$ & $152 \pm 0.1$ & $153 \pm 0.1$ & $152 \pm 0.1$ \\
\hline Body mass index $\left(\mathrm{kg} / \mathrm{m}^{2}\right)$ & $27.9 \pm 4.0$ & $27.7 \pm 3.6$ & $27.9 \pm 3.9$ & $27.8 \pm 3.5$ \\
\hline Abdomen $(\mathrm{cm})$ & $95.7 \pm 11.9$ & $96.8 \pm 7.5$ & $94.1 \pm 11.2$ & $94.1 \pm 6.9$ \\
\hline Waist $(\mathrm{cm})$ & $85.2 \pm 10.7$ & $86.4 \pm 8.6$ & $84.7 \pm 9.1$ & $84.7 \pm 7.5$ \\
\hline \%G (body fat percentage) & $40.9 \pm 2.7$ & $40.0 \pm 1.9$ & $39.0 \pm 2.7$ & $39.0 \pm 2.1$ \\
\hline
\end{tabular}

Note: Data are presented as mean \pm standard deviation. 


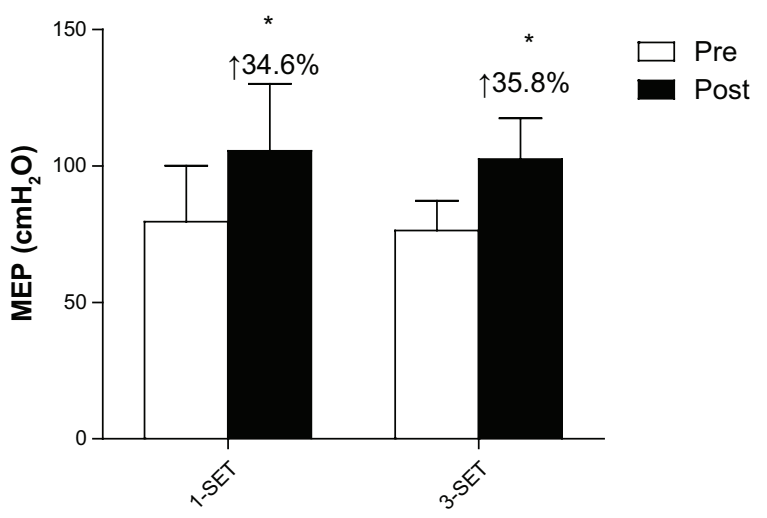

Figure 2 Maximum expiratory pressure (MEP) before and after 24 sessions of resistance training.

Notes: Data are presented as means \pm standard deviation. Percent differences between pre-test and post-test. $* P<0.05$, versus pre-test.

Abbreviations: I-SET, single set; 3-SET, three sets.

post: $102.5 \pm 14.9 \mathrm{cmH}_{2} \mathrm{O}$; effect size: 2.40 ), respectively (Figure 2).

MIP also increased significantly $(P<0.05)$ in both groups (pre: $90.0 \pm 17.0 \mathrm{cmH}_{2} \mathrm{O}$; post: $102.3 \pm 17.5 \mathrm{cmH}_{2} \mathrm{O}$; effect size: 0.71 ) and (pre: $72.5 \pm 16.0 \mathrm{cmH}_{2} \mathrm{O}$; post: $80.6 \pm 20.1 \mathrm{cmH}_{2} \mathrm{O}$; effect size: 0.51 ) for the 1-SET and 3-SET groups, respectively (Figure 3).

Both the 1-SET and 3-SET groups significantly improved $(P<0.05)$ in the sit-to-stand test (pre: $19.0 \pm 3.2$ repetitions; post: $21.0 \pm 3.8$ repetitions; effect size: 0.61 ) and (pre: $18.6 \pm 3.0$ repetitions; post: $21.6 \pm 2.9$ repetitions; effect size: 1.00 ), respectively (Figure 4).

In the exercises assessed, both groups demonstrated significantly $(P<0.0001)$ increased levels of muscle strength (40\%-80\%) after training (Table 3). The intergroup comparison did not show any significant difference in any of the analyzed parameters.

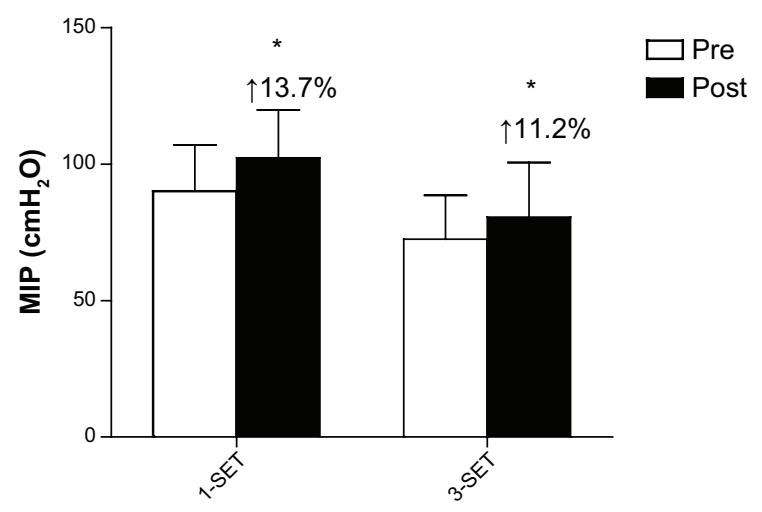

Figure 3 Maximum inspiratory pressure (MIP) before and after 24 sessions of resistance training.

Notes: Data are presented as means \pm standard deviation. Percent differences between pre-test and post-test. $* P<0.05$, versus pre-test.

Abbreviations: I-SET, single set; 3-SET, three sets.

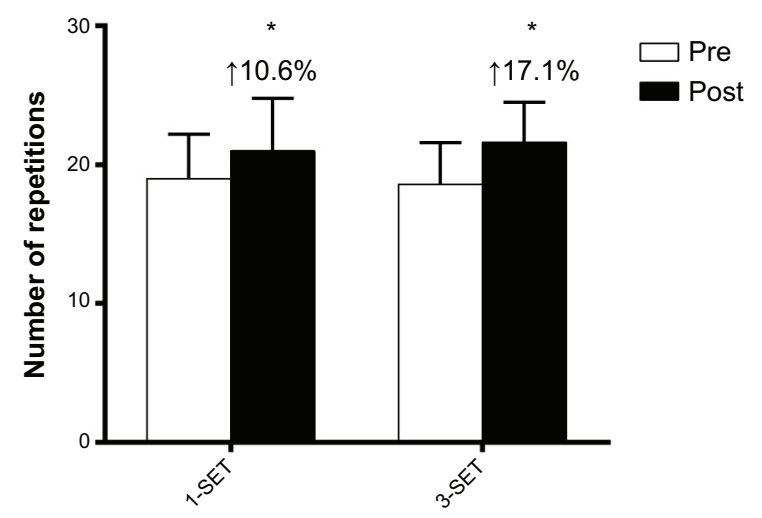

Figure 4 Functional performance in the sit-to-stand test before and after 24 sessions of resistance training.

Notes: Data are presented as means \pm standard deviation. Percent differences between pre-test and post-test. $* P<0.05$, versus pre-test.

Abbreviations: I-SET, single set; 3-SET, three sets.

\section{Discussion}

The main finding of this study was that single set (SS) and multiple set (MS) RT programs are efficient methods for improving performance in the sit-to-stand test and increasing muscle strength, MIP, and MEP in elderly women after 24 sessions of training. Both the 1-SET and 3-SET groups showed statistically significant changes in respiratory muscle strength. To the best of our knowledge, this was the first study to compare the effects of RT volume on respiratory muscle strength in elderly women.

Currently, MIP and MEP are widely used, and the objectives are to identify possible weakness, respiratory muscle failure, and fatigue, as well as to quantify the effects of respiratory training. ${ }^{6-10}$ This capacity is considered as an important parameter for the assessment, mainly in individuals with certain chronic diseases, and the elderly, because they experience significant respiratory changes with the onset of senescence. ${ }^{8,9,18}$

Respiratory muscle strength may have increased because, during RT, abdominal muscles are used as stabilizers, through isometric contractions that maintain body posture, contributing to improved muscular strength. ${ }^{19}$ Among the different exercises used in our protocol (submaximal/ maximal strength between 8-12 MR), the deadlift is considered an important exercise that encourages the activation of important respiratory muscles, namely, the rectus abdominis, transverse abdominis, and serratus anterior. ${ }^{19}$ Additionally, abdominal exercises are normally prescribed in RT programs ${ }^{17}$ Hackett et al ${ }^{11}$ suggest that other potential factors which may contribute to the increase of the respiratory muscles strength include the involvement of the diaphragm during RT. 
Table 3 Repetition test ( $10 \mathrm{MR}$ ) before and after 24 sessions of training in the group performing a single set (I-SET, $\mathrm{n}=\mathrm{I}$ I) and in the group performing three sets (3-SET, $\mathrm{n}=8$ )

\begin{tabular}{|c|c|c|c|c|c|c|c|}
\hline $\begin{array}{l}\text { Exercises } \\
\text { in } \mathbf{~ k g}\end{array}$ & Group & $\begin{array}{l}\text { Pre } \\
(\text { mean } \pm \text { SD) }\end{array}$ & $\begin{array}{l}\text { Post } \\
\text { (mean } \pm \text { SD) }\end{array}$ & $\begin{array}{l}\Delta \\
(\text { mean } \pm \text { SD })\end{array}$ & $\begin{array}{l}\Delta \% \\
(\text { mean } \pm \text { SD })\end{array}$ & $P$-value & $\begin{array}{l}\text { Effect } \\
\text { size }\end{array}$ \\
\hline \multirow[t]{2}{*}{ Bench press } & I-SET & $\mid 3.8 \pm 1.7$ & $21.6 \pm 3.2$ & $7.8 \pm 2.1$ & $56.5 \pm 13.8$ & $<0.000 I^{*}$ & 4.59 \\
\hline & 3-SET & $14.3 \pm 3.5$ & $22.0 \pm 4.0$ & $7.8 \pm 2.0$ & $57.5 \pm 21.0$ & $<0.000 I^{*}$ & 2.20 \\
\hline \multirow[t]{2}{*}{ Deadlift } & I-SET & $10.0 \pm 3.8$ & $16.5 \pm 3.6$ & $6.5 \pm 2.4$ & $60.0 \pm 23.5$ & $<0.000 I^{*}$ & 1.71 \\
\hline & 3-SET & $13.3 \pm 3.8$ & $20.5 \pm 4.1$ & $7.3 \pm 1.5$ & $73.2 \pm 28.8$ & $<0.000 I^{*}$ & 1.89 \\
\hline \multirow[t]{2}{*}{ Rowing } & I-SET & $19.5 \pm 2.7$ & $26.6 \pm 2.5$ & $7.1 \pm 3.7$ & $38.3 \pm 20.4$ & $<0.000 I^{*}$ & 2.63 \\
\hline & 3-SET & $16.9 \pm 2.6$ & $26.5 \pm 2.6$ & $9.6 \pm 2.1$ & $58.8 \pm 17.8$ & $<0.000 I^{*}$ & 3.69 \\
\hline \multirow[t]{2}{*}{ Calf raise } & I-SET & $21.8 \pm 3.5$ & $38.6 \pm 2.3$ & $16.8 \pm 3.4$ & $79.1 \pm 22.0$ & $<0.000 I^{*}$ & 4.80 \\
\hline & 3-SET & $25.0 \pm 2.8$ & $40.6 \pm 8.6$ & $15.6 \pm 5.6$ & $66.1 \pm 28.5$ & $<0.000 I^{*}$ & 5.57 \\
\hline
\end{tabular}

Notes: $* P \leq 0.001$, versus pretest; $\Delta \%=$ average percent difference between pre- and post-tests. Data are presented as mean \pm standard deviation.

Abbreviations: MR, maximum repetitions, SD, standard deviation.

Watsford and Murphy ${ }^{20}$ demonstrated a significant increase in respiratory muscle strength in elderly women participating in specific respiratory muscle training over a period of 8 weeks. Other benefits observed included a reduction in the submaximal heart rate and an increase in maximum voluntary ventilation, when compared to a control group. A study ${ }^{12}$ investigated the effects of flexibility training (proprioceptive neuromuscular facilitation) combined with resistance training (elastic resistance bands) on respiratory muscle strength in adult women, and its results showed significant increases in MIP and MEP. In accordance with our results, these studies suggested that specific respiratory muscle training and resistance training are important adjuvants in improving respiratory muscle strength. ${ }^{11,12,20}$

In a recent study, MIP and MEP were observed to decrease according to the fragility of the elderly person. In addition, inspiratory and expiratory muscle strengths were positively correlated with general muscle strength. ${ }^{21}$ These data justify the need for further studies to assess the effects of resistance exercise on inspiratory and expiratory muscle strength, with the aim of minimizing or reversing loss of respiratory function, given that a reduction in pulmonary function is associated with an increase in morbidity and mortality rates..$^{18,22,23}$

Another important benefit observed in this study was muscular strength gains. This physical component is related to increased mobility and walking pace, due to increased musculoskeletal excitability and muscle innervation, leading to greater functional independence. ${ }^{3,24,25}$ Furthermore, increased muscle strength can contribute to a reduced incidence of falls, thus preventing bone fractures. Therefore, muscle strength is an important physical component for maintaining the health of the elderly. ${ }^{2,26}$
The elderly women who participated in this study demonstrated statistically significant increases in levels of muscle strength after 24 sessions of progressive RT over a period of 12 weeks, without significant intergroup differences (1-SET versus 3 -SET). These results could be explained by the short training period (neural adaptations) used in agreement with a recent study. ${ }^{27}$ Galvão and Taaffe ${ }^{28}$ assessed the effects of SS and MS RT in the elderly, and their results demonstrated that after 20 weeks of training, both groups significantly increased their strength in the seven different types of exercises analyzed. These results corroborate a meta-analysis published by Fröhlich et $\mathrm{a}^{29}$ which demonstrated that SS and MS RT can guarantee a similar strength increase in individuals using short training periods, but the benefits of using MS RT increase with longer training periods (over 25 weeks). Another meta-analysis ${ }^{30}$ reported that when subjects used two to three sets per exercise, they demonstrated a $46 \%$ increase in strength, in comparison with a single set, regardless of the training level or the program duration. The difference between these meta-analysis results could be related to the inclusion methods employed by the different studies and their statistical delineation.

The results of this study showed significant increases in the sit-to-stand test. Currently, this test is considered an important indicator of lower limb strength, and functional capacity to perform basic activities in older adult women, ${ }^{13,31-33}$ in addition to its correlation with quadriceps strength and lean mass. ${ }^{33}$ The findings from this study corroborate those of Galvão and Taaffe, ${ }^{28}$ in identifying that SS programs performed two times per week are able to improve performance in the sit-to-stand test.

No significant differences were observed in anthropometric indexes between the groups after 24 training sessions. These results can be explained by the absence of nutritional 
intervention, given that the women maintained their normal eating habits during the study period. Washburn et $\mathrm{al}^{34}$ assessed the effects of SS RT on body composition using double-emission densitometry in overweight adults. The exercise protocol comprised a single set of nine exercises, performed three times per week at 3-6 MR intensity over a period of 6 months. The results revealed that, compared to the control group, the intervention group had a significant increase in lean mass. The intervention group did not show any change in body fat, whereas the control group showed a significant increase in body fat (mean: $1.9 \%$ ). There was no nutritional intervention in these groups, and the minimum training volume helped to maintain a stable body fat mass. ${ }^{34}$ That evidence corroborates our study, given that both groups received no nutritional intervention and showed no significant changes in their body fat percentages.

\section{Conclusion}

Single- and multiple-set resistance training programs increased respiratory muscle strength, muscle strength, and sit-to-stand test performance in elderly women after 24 sessions of training. Elderly women who do not prioritize time for physical activity or do not have a good adherence to regular programs should be recommended to start with single-set resistance training programs as a short-term strategy.

\section{Acknowledgments}

The authors would like to acknowledge Coordenação de Aperfeiçoamento de Pessoal de Nível Superior (CAPES) and Fundação de Apoio à Pesquisa e Inovação Tecnológica do Estado de Sergipe (FAPITEC/SE) for their funding support for this study. We also thank teacher Abilio Borghi for assistance with the grammar review of the manuscript.

\section{Disclosure}

The authors report no conflicts of interest in this work.

\section{References}

1. World Health Organization. Interesting facts about ageing [Internet] Geneva: WHO; 2012 [cited 2013 Aug 29]. Available from: http://www. who.int/ageing/about/facts/en/index.html. Accessed April 12, 2014.

2. Westcott WL. Resistance training is medicine: effects of strength training on health. Curr Sports Med Rep. 2012;11(4):209-216.

3. Fiatarone MA, Marks EC, Ryan ND, Meredith CN, Lipsitz LA, Evans WJ. High-intensity strength training in nonagenarians. Effects on skeletal muscle. JAMA. 1990;263(22):3029-3034.

4. Vaz Fragoso CA, Enright PL, McAvay G, Van Ness PH, Gill TM. Frailty and respiratory impairment in older persons. Am J Med. 2012; 125(1):79-86.

5. Sin DD, Wu L, Man SF. The relationship between reduced lung function and cardiovascular mortality: a population-based study and a systematic review of the literature. Chest. 2005;127(6):1952-1959.
6. American Thoracic Society/European Respiratory Society. ATS/ERS Statement on respiratory muscle testing. Am J Respir Crit Care Med. 2002;166(4):518-624.

7. Neder JA, Andreoni S, Castelo-Filhop A, Nery LE. Reference values for lung function tests. I. Static volumes. Braz J Med Biol Res. 1999; 32(6):703-717.

8. Kim J, Sapienza CM. Implications of expiratory muscle strength training for rehabilitation of the elderly: Tutorial. J Rehabil Res Dev. 2005; 42(2):211-224.

9. Simões RP, Deus AP, Auad MA, Dionísio J, Mazzonetto M, BorghiSilva A. Maximal respiratory pressure in healthy 20 to 89 year-old sedentary individuals of central São Paulo State. Rev Braz Fisioter. 2010;14(1):60-67.

10. HajGhanbari B, Yamabayashi C, Buna TR, et al. Effects of respiratory muscle training on performance in athletes: a systematic review with meta-analyses. J Strength Cond Res. 2013;27(6):1643-1663.

11. Hackett DA, Johnson NA, Chow CM. High-volume resistance training session acutely diminishes respiratory muscle strength. $J$ Sports $S c i$ Med. 2012;11(1):26-30.

12. Areas GP, Borghi-Silva A, Lobato AN, Silva AA, Freire RC Jr, Areas FZ. Effect of upper extremity proprioceptive neuromuscular facilitation combined with elastic resistance bands on respiratory muscle strength: a randomized controlled trial. Braz J Phys Ther. 2013;17(6):541-546.

13. Rikli RE, Jones CJ. Development and validation of a functional fitness test for community-residing older adults. J Aging Phys Act. 1999; 7(2):129-161.

14. Durnin JV, Womersley J. Body fat assessed from total body density and its estimation from skinfold thickness: measurements on 481 men and women aged from 16 to 72 years. Br J Nutr. 1974;32(1):77-97.

15. Siri WE. Body composition from fluid spaces and density: analysis of methods. 1961. Nutrition. 1993;9(5):480-491.

16. Norton K, Olds T. Antropométrica. Porto Alegre, Brazil: Artmed; 2005.

17. American College of Sports Medicine, Chodzko-Zajko WJ, Proctor DN, Fiatarone Singh MA, et al. American College of Sport Medicine position stand. Exercise and physical activity for older adults. Med Sci Sports Exerc. 2009;41(7):1510-1530.

18. Phillips WT, Benton MJ, Wagner CL, Riley C. The effect of single set resistance training on strength and functional fitness in pulmonary rehabilitation patients. J Cardiopulm Rehabil. 2006;26(5):330-337.

19. Martuscello JM, Nuzzo JL, Ashley CD, Campbell BI, Orriola JJ, Mayer JM. Systematic review of core muscle activity during physical fitness exercises. J Strength Cond Res. 2013;27(6):1684-1698.

20. Watsford M, Murphy A. The effects of respiratory-muscle training on exercise in older women. J Aging Phys Act. 2008;16(3):245-260.

21. Pegorari MS, Ruas G, Patrizzi LJ. Relationship between frailty and respiratory function in the community-dwelling elderly. Braz J Phys Ther. 2013;17(1):9-16.

22. Santana H, Zoico E, Turcato E, et al. Relation between body composition, fat distribution, and lung function in elderly men. Am J Clin Nutr. 2001;73(4):827-831.

23. Sin DD, Wu L, Man SF. The relationship between reduced lung function and cardiovascular mortality: a population-based study and a systematic review of the literature. Chest. 2005;127(6):1952-1959.

24. Bean JF, Kiely DK, Herman S, et al. The relationship between leg power and physical performance in mobility-limited older people. $J \mathrm{Am}$ Geriatr Soc. 2002;50(3):461-467.

25. Kalyani RR, Tra Y, Yeh HC, Egan JM, Ferrucci L, Brancati FL. Quadriceps strength, quadriceps power, and gait speed in older U.S. adults with diabetes mellitus: results from the National Health and Nutrition Examination Survey, 1999-2002. J Am Geriatr Soc. 2013;61(5):769-775.

26. Marin RV, Pedrosa MA, Moreira-Pfrimer LD, Matsudo SM, LazarettiCastro M. Association between lean mass and handgrip strength with bone mineral density in physically active postmenopausal women. J Clin Densitom. 2010;13(1):96-101.

27. Radaelli R, Botton CE, Wilhelm EN, et al. Low- and high-volume strength training induces similar neuromuscular improvements in muscle quality in elderly women. Exp Gerontol. 2013;48(8):710-716. 
28. Galvão DA, Taaffe DR. Resistance exercise dosage in older adults: single-versus multiset on physical performance and body composition. J Am Geriatr Soc. 2005;53(12):2090-2097.

29. Fröhlich M, Emrich E, Schmidtbleicher D. Outcome effects of singleset versus multiple-set training - an advanced replication study. Res Sports Med. 2010;18(3):157-175.

30. Krieger JW. Single versus multiple sets of resistance exercise: a metaregression. J Strength Cond Res. 2009;23(6):1890-1901.

31. Santos RG, Tribess S, Meneguci J, et al. [Lower limb strength as an indicator of functional disability in older individuals]. Motriz. 2013;19(3):S35-S42. Portuguese.
32. McCarthy EK, Horval MA, Holtsberg PA, Wisenbaker JM. Repeated chair stands as a measure of lower limb strength in sexagenarian women. J Geront A Biol Sci Med Sci. 2004;59(11):1207-1212.

33. Taaffe DR, Duret C, Wheeler S, Marcus R. Once-weekly resistance exercise improves muscle strength and neuromuscular performance in older adults. J Am Geriatr Soc. 1999;47(10):1208-1214.

34. Washburn RA, Kirk EP, Smith BK, et al. One set resistance training: effect on body composition in overweight young adults. J Sports Med Phys Fitness. 2012;52(3):273-279.

\section{Publish your work in this journal}

Clinical Interventions in Aging is an international, peer-reviewed journal focusing on evidence-based reports on the value or lack thereof of treatments intended to prevent or delay the onset of maladaptive correlates of aging in human beings. This journal is indexed on PubMed Central, MedLine,
CAS, Scopus and the Elsevier Bibliographic databases. The manuscript management system is completely online and includes a very quick and fair peer-review system, which is all easy to use. Visit http://www.dovepress. com/testimonials.php to read real quotes from published authors. 